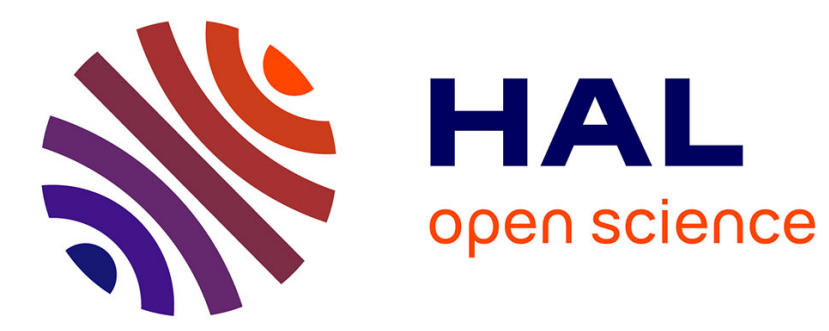

\title{
Strong current actions on ferrimagnetic domain walls in the creep regime
}

E Haltz, J. Sampaio, R. Weil, Y. Dumont, A. Mougin

\section{To cite this version:}

E Haltz, J. Sampaio, R. Weil, Y. Dumont, A. Mougin. Strong current actions on ferrimagnetic domain walls in the creep regime. Physical Review B: Condensed Matter and Materials Physics (1998-2015), 2019, 99, 10.1103/PhysRevB.99.104413 . hal-02331717

\section{HAL Id: hal-02331717 https://hal.science/hal-02331717}

Submitted on 24 Oct 2019

HAL is a multi-disciplinary open access archive for the deposit and dissemination of scientific research documents, whether they are published or not. The documents may come from teaching and research institutions in France or abroad, or from public or private research centers.
L'archive ouverte pluridisciplinaire HAL, est destinée au dépôt et à la diffusion de documents scientifiques de niveau recherche, publiés ou non, émanant des établissements d'enseignement et de recherche français ou étrangers, des laboratoires publics ou privés. 


\title{
Strong current actions on ferrimagnetic domain walls in the creep regime
}

\author{
E. Haltz, ${ }^{1}$ J. Sampaio, ${ }^{1}$ R. Weil,,${ }^{1}$ Y. Dumont,${ }^{2}$ and A. Mougin ${ }^{1, *}$ \\ ${ }^{1}$ Laboratoire de Physique des Solides, CNRS, Univ. Paris-Sud, Université Paris-Saclay, Bât. 510, 91405 Orsay, France \\ ${ }^{2}$ GEMaC, CNRS-UVSQ Batiment Fermat, 45 avenue des Etats-Unis, 78035 Versailles, France
}

(Received 13 June 2018; revised manuscript received 11 January 2019; published 11 March 2019)

\begin{abstract}
We observe domain-wall (DW) motion in ferrimagnetic TbFe wires with perpendicular anisotropy under combined field and current in the creep regime. The current action on the DW is double: Joule heating and spin-transfer torque. We propose a genuinely robust analysis of velocity, separating thermal effects and spin-transfer torque, quantifying the latter as an equivalent field in the so-called one-dimensional (1D) model. Its efficiency is much larger than in transition-metal ferromagnets above room temperature. The equivalent field reveals the large polarization-to-magnetization ratio in ferrimagnets despite a vanishing $M_{s}$. The usual 1D DW model is extended to mimic creep and predicts that, in low net magnetization systems, the internal DW structure precesses with currents above a field-independent threshold, leading to two propagation regimes with different mobilities. This is another example of how the internal DW magnetization is relevant in creep. We could not detect experimentally these two regimes, possibly because of the dispersion of the data.
\end{abstract}

DOI: 10.1103/PhysRevB.99.104413

Spintronic nanodevices that store and process nonvolatile information are based on the magnetic reversal of nanomagnets [1,2]. Experimental results from field-driven as well as current-driven switching show that the reversal is mediated by domain-wall (DW) propagation, even in nanomagnets of just a few tens of nm [3-5]. The main mechanism for currentinduced DW motion (DWM) is the relaxation of a spin current in the magnetic structure. If the current is polarized inside the magnetic material, we speak about spin-transfer torque (STT). The efficiency of the polarized current action on a magnetic structure is expressed by a drift speed [6]:

$$
u=\frac{g \mu_{B} P}{2 e M_{S}} J
$$

where $P$ is the spin polarization of current of density $J$ in the magnetic media, $M_{S}$ is the total (effective) magnetization of this media, $g$ is the (effective) Lande factor, $\mu_{B}$ is the Bohr magneton, and $e$ is the electron charge. In the classical ferromagnetic materials that have been studied, $P$ and $M_{S}$ share the same physical origin and so the same dependence on temperature, and therefore the ratio $P / M_{s}$ which governs the efficiency of STT is fixed [7]. To increase this quantity, a good solution is to focus on more exotic materials such as ferrimagnetic alloys. Equation (1) was developed for ferromagnets but it holds for ferrimagnetic alloys by using effective parameters away from compensation points (magnetic or angular). Rareearth-transition-metal (RE-TM) ferrimagnetic alloys, such as TbFe, have two populations of magnetic moments that are antiferromagnetically coupled [8,9]. One population is the spin-polarized valence electrons of the RE and of the TM [8,9] and the second one is the localized $4 f$ electrons of RE. Even if current polarization is due to the first one, the net magnetization of this alloy $M_{s}$ is the sum of moments of

\footnotetext{
*alexandra.mougin@u-psud.fr
}

the two populations. That is why ferrimagnetic materials can exhibit a vanishingly small magnetization and yet a sizable spin polarization, both tunable by acting on the composition of alloys or by changing the temperature [8,10,11]. A few results exist showing clear DWM under current in ferrimagnets [12-14].

In our case, we observe DWM in the creep regime. The study of the creep regime in current-driven DWs lead to nonconsensual interpretations [15-17]. One of the challenges is to account fully for Joule heating in a dynamical regime that relies on thermal activation [18]. We propose the first rigorous study of DW creep motion in TbFe ferrimagnets under current. Moreover, in the usual creep theory, the dynamics are assumed to be independent of the internal structure of the DW. However, recently, it was shown that the DzyaloshinskiiMoriya interaction or the in-plane magnetic field affects the DW velocity in complex ways that can only be understood by considering the details of the DW structure [17,19]. So far, dynamic changes of the DW structure were never considered in the creep analysis. We propose a model in which the dynamics of the DW structure are relevant also in the creep.

The studied film is $7 \mathrm{~nm}$ of the amorphous alloy $\mathrm{Tb}_{21} \mathrm{Fe}_{79}$ with $5 \mathrm{~nm} \mathrm{Al}$ cover with perpendicular magnetic anisotropy deposited by co-evaporation on a $\mathrm{Si} / \mathrm{SiO}_{x}(500 \mathrm{~nm})$ substrate in ultrahigh vacuum. Details of growth and basic properties of the stacks are published elsewhere [20]. The film was characterized by using the magneto-optic Kerr effect (MOKE) and the anomalous Hall effect (AHE) resistivity measurements which are mainly proportional to spin polarization of electrons at the Fermi level (equivalent to $P$ ) $[8,10]$. The net magnetization $M_{s}$ was measured by vibrating sample magnetometry (VSM). The polarization and magnetization results are shown in Fig. 1(a) as a function of temperature. The magnetic compensation temperature $\left(M_{s}=0\right)$ is well below the smallest experimental temperature $(50 \mathrm{~K})$ and was not reached. The Curie temperature is $375 \mathrm{~K}$. Despite being 


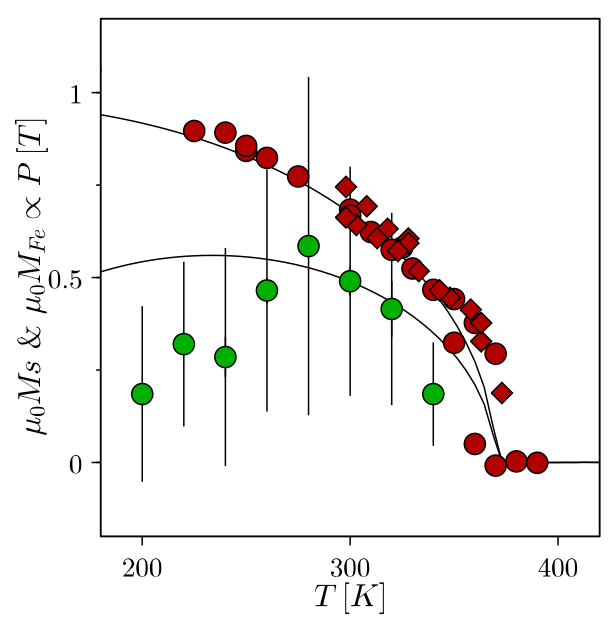

FIG. 1. Magnetic properties of TbFe film in temperature: net magnetization $M_{s}$ obtained from VSM hysteresis loop amplitude (green circles), MOKE hysteresis loops (red diamonds), and Hall resistivity (red circles) amplitudes in arbitrary units chosen so they superimpose. The continuous lines are the result of mean-field calculations.

quite noisy ( $\mu_{0} M_{s} \approx 0.3 \mathrm{~T}$ at room temperature), $M_{s}$ exhibits a thermal evolution obviously different from that of $P$. We performed a mean-field calculation of coupled RE and TM sublattice magnetizations (following Refs. [10,21]). TM magnetization was adjusted to match the temperature evolution of the AHE and MOKE data. The net magnetization curve superimposed "naturally" on the experimental VSM data. The main feature extracted from Fig. 1 is that $P / M_{s}$ is larger than in usual ferromagnets.

To inject large current density, the sample is structured in tracks of $5 \mu \mathrm{m}$ by electron-beam lithography and $\mathrm{Ar}^{+}$ ion-beam etching. Contacts made of $\operatorname{Ti}(5 \mathrm{~nm}) / \mathrm{Au}(50 \mathrm{~nm})$ are deposited and connected to the experimental setup. The sample is fixed on a heater with temperature control. To observe directly the DWM, we use a commercial Kerr microscope [22].

To determine the dynamic regime of these DWs, we first measure the velocity under continuous field (without current, $J=0$ ) at different temperatures $T$, shown in Fig. 2(a). We observe a nonlinear behavior of velocity versus field and a strong dependence with $T$. This DW dynamics is characteristic of a creep regime with $\ln (v) \propto H^{-1 / 4}$. Indeed, in this regime, the DWM occurs by discrete hopping of the DW between pinned configurations due to weak pinning centers [18]; the motion is induced by thermal activation and favored by the field (below the depinning field $H_{\text {dep }}$ ). The velocity of DWs [Eq. (2)] is described by an Arrhenius law with $v_{0}$ a prefactor homogenous to a speed, $k_{B}$ the Boltzmann constant, $k_{B} T_{\text {dep }}$ the pinning barrier amplitude, and $\Delta E$ the energy barrier:

$$
v(T, H)=v_{0} e^{\left(-\frac{\Delta E}{k_{B} T}\right)}, \quad \Delta E=k_{B} T_{\mathrm{dep}}\left[\left(\frac{H}{H_{\mathrm{dep}}}\right)^{-1 / 4}-1\right] .
$$

All three parameters $\left(v_{0}, T_{\text {dep }}\right.$, and $\left.H_{\text {dep }}\right)$ are temperature dependent whatever the material, as demonstrated in Ref. [18]. In our sample, the impact of temperature is
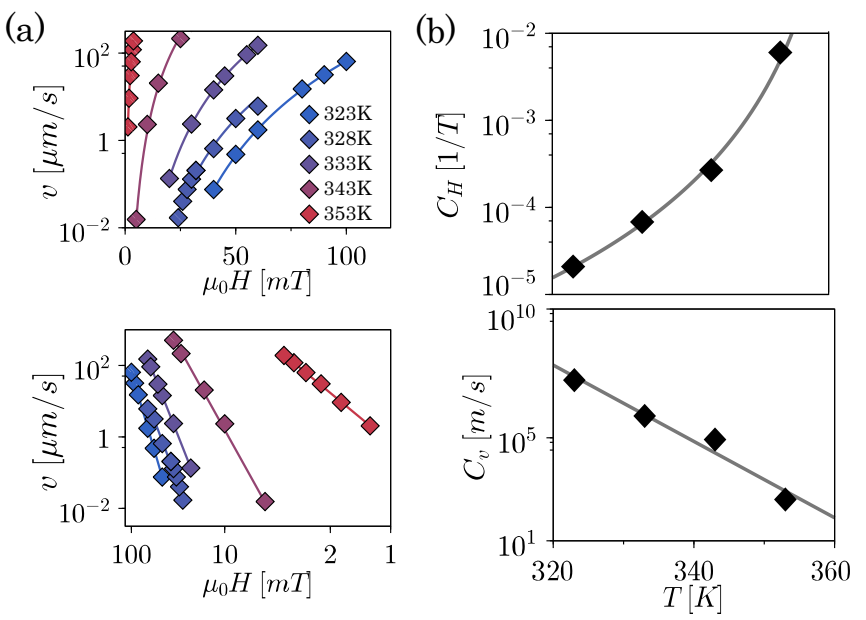

FIG. 2. (a) DW velocities on a logarithmic scale as a function of magnetic field: linear representation (top) and $H^{-1 / 4}$ scale (bottom) at different $T_{s p}$ indicated in the figure with $J=0$. Continuous lines are fits using the Eq. (3) signature of creep. (b) Creep parameters $C_{H}(T)$ and $C_{V}(T)(J=0)$. The solid lines are polynomial fits used in the following fitting process.

exacerbated by the strong dependence of its magnetic properties. Since $H_{\text {dep }}$ is not reached experimentally, we cannot determine independently all three creep parameters. With $C_{V}=v_{0} \exp \left(\frac{T_{\text {dep }}}{T}\right)$ and $C_{H}=\frac{1}{H_{\text {dep }}}\left(\frac{T}{T_{\text {dep }}}\right)^{4}$, we can use a compact creep law [Eq. (3)] to adjust our experimental velocities versus field in temperature:

$$
v(T, H)=C_{V} e^{-\left(C_{H} H\right)^{-1 / 4}} .
$$

The temperature-dependent creep parameters $C_{V}(T)$ (units of velocity) and $C_{H}(T)$ (units of the inverse of field) obtained from the velocities fitting show a large variation with temperature [Fig. 2(b)].

We next measured DW motion induced by the combined effects of current pulses superimposed with continuous magnetic field, with a temperature set point $T_{s p}=328 \mathrm{~K}$. Current pulses of width $t_{p w}=10 \mu \mathrm{s}$ with a period $t_{p p}=100 \mu \mathrm{s}$ were applied to a track with two DWs. Figure 3(a) shows illustrative Kerr images. All measurements were repeated for two current directions, up or down central domain magnetization, and + or - field. For each configuration of $H$ and $J$, we distinguish a fast and a slow DW ( $v_{\text {fast }}$ and $\left.v_{\text {slow }}\right)$. If the current is reversed, the fast DW becomes the slow, and vice versa [as shown in the four first images in Fig. 3(a)]. If the current density is increased ( 1 versus 5), $v_{\text {fast }}-v_{\text {slow }}$ increases, but also $v_{\text {fast }}$ and $v_{\text {slow }}$. If the applied field is increased (1 versus 6), both $v_{\text {fast }}$ and $v_{\text {slow }}$ increase, but also $v_{\text {fast }}-v_{\text {slow }}$. Actually, with these measurements, it is possible to directly observe the two main effects of current on DWM. First Joule heating modifies the creep dynamics and makes DWM easier in both directions. This is why in 1 versus 5, both DW were faster. Second, a STT-like action pushes DWs along the electron flow and adds (or subtracts) to the field action. This is why in 1 versus 5 , the difference $v_{\text {fast }}-v_{\text {slow }}$ is larger. From 1 versus 6, both velocities increase but the fact that $v_{\text {fast }}-v_{\text {slow }}$ increases as well demonstrates the nonlinearity of velocity versus current and field. If the applied magnetic field is decreased and 
(a)

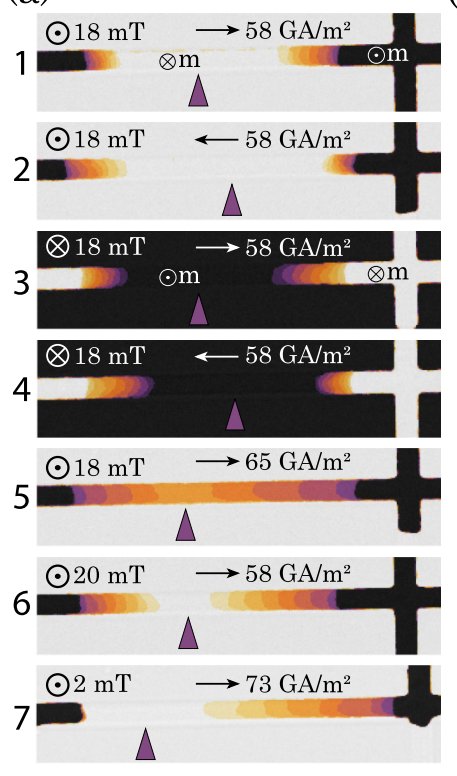

(b)
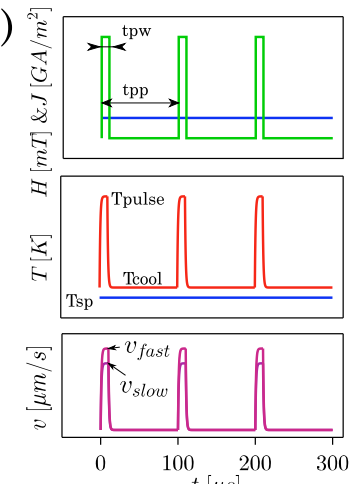

(c)

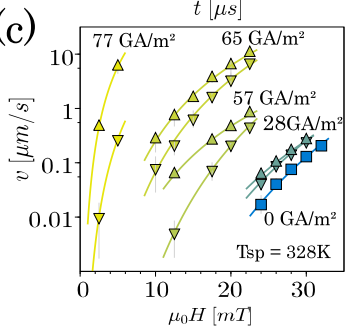

FIG. 3. (a) Illustrative superposition of Kerr images (taken every $1 \mathrm{~s})$ at $T_{s p}=328 \mathrm{~K}$ for field and current indicated in each numbered picture. Dark (bright) regions correspond to up (down) magnetization. A sequence of magnetic fields was used to nucleate and drive two DWs to the initial configuration. The following images appear with an orange contrast. The difference between $v_{\text {fast }}$ and $v_{\text {slow }}$ is illustrated by the center of the remaining central domain (marked by a triangle in the images). (b) Time line of the experiments and of the corresponding parameters (c) Velocity ( $v_{\text {fast }}$ up triangles, $v_{\text {slow }}$ down triangles) versus $H$ at different $J$ at $T_{s p}=328 \mathrm{~K}$. Continuous lines are fits using the creep law given by Eq. (4).

the current increased ( 1 versus 7$)$, both effects are larger. Figure 3(c) shows velocities as a function of $H$ for a few values of $J$. For all the configurations with the same $|H|$ and $|J|$, all $v_{\text {fast }}$ are approximately the same (as well as all $v_{\text {slow }}$ ) and the average value is shown for clarity. For a given $(H, J)$, two points are depicted but each pair is the results of eight independent experiments $( \pm H, \pm J$, up-down, and down-up walls).

To quantify the current actions, we consider a field $H_{e q}$ equivalent to the STT-like action on the DW. This $H_{e q}$ is the field necessary to induce the same macroscopic DW motion as a given current density. This definition of $H_{e q}$ is quite common in the so-called 1D model for free DW (without pinning), as reminded in the Supplemental Material [23]. Our model does not include any $J^{2}$ STT-like effects since such a contribution cannot be used to move DWs directionally and we focus on directional control of DWM. Being in the creep requires a precise knowledge of temperature. We determined the temperature time profile by using pulses of variable duration (see Appendix). The heating time is about $2.5 \mu \mathrm{s}$, shorter than $t_{p w}$. That is why we approximate the temperature time profile by two constant temperatures, $T_{\text {pulse }}$ during the current pulse and $T_{\text {cool }}$ afterwards [Fig. 3(b)], both dependent on $J$. We can then consider that the measured velocity is an average of the velocity during the current pulse, of duration $t_{p w}$ with the current-induced $H_{e q}$ and temperature $T_{\text {pulse }}$, and the velocity during the period between pulses, of duration $t_{p p}-t_{p w}$, with

temperature $T_{\text {cool }}$ and no field-induced $H_{e q}$. Therefore, for each value of $|J|$ and each of four configurations ( $\pm H$, updown, and down-up walls), we fit the measured velocities with the compact creep law [Eq. (4)]:

$$
\begin{aligned}
\langle v\rangle(H, J)= & \frac{t_{p w}}{t_{p p}} C_{V}\left(T_{\text {pulse }}\right) e^{-\left[C_{H}\left(T_{\text {pulse }}\right)\left(H \pm H_{e q}\right)\right]^{-1 / 4}} \\
& +\frac{t_{p p}-t_{p w}}{t_{p p}} C_{V}\left(T_{\text {cool }}\right) e^{-\left[C_{H}\left(T_{\text {cool }}\right) H\right]^{-1 / 4}} .
\end{aligned}
$$

In this model, the sign \pm before $H_{e q}$ corresponds to current along or opposed the field-induced propagation ( + for fast DWs and - for slow DWs). Because the evolution of the creep parameters with temperature is known [Fig. 2(b)], the fitting procedure only has three fitting parameters (per value of $|J|): T_{\text {pulse }}, T_{\text {cool }}$, and $H_{e q}$. We have assumed two reasonable bounding conditions: that $T_{\text {pulse }}>T_{\text {cool }}>T_{s p}$ (within a $1 \mathrm{~K}$ tolerance) and that the heating amplitude, $\Delta T=T_{\text {pulse }}-T_{\text {cool }}$, follows within a $50 \%$ margin an independently determined Joule heating law $\Delta T=A J^{2}$ (described in the Appendix). This last bounding condition is very loose and does not bind the fit parameters nor their evolution with $J$ except at very low currents, where it leads to a reduced dispersion of the fit parameters and imposes $T_{\text {pulse }}-T_{\text {cool }} \rightarrow 0$ when $J \rightarrow 0$. The simultaneous fits of $v_{\text {fast }}$ and $v_{\text {slow }}$ are solid lines in Fig. 3(c). The temperatures $T_{\text {pulse }}, T_{\text {cool }}$, and the equivalent field $H_{e q}$ are shown Fig. 4.

We first comment on the temperatures deduced from the fits. First, for large current values, the velocity during the current pulse dominates the domain-wall velocity: because $T_{\text {pulse }} \gg T_{\text {cool }}$, the temperature between the pulse does not affect the measured velocity. Therefore, the fitted $T_{\text {cool }}$ temperature at high $J$ is neither accurate nor relevant and may deviate from $T_{s}$ by a few degrees. On the contrary, the values obtained for $T_{\text {cool }}$ are very close to $T_{s p}$ for small $J$. They are both accurate and relevant. Second, a variation of several tens of degrees is evidenced in $T_{\text {pulse }}$. An independent determination of the amplitude of Joule heating vs $J$ was performed. We measured the coercive field under different current densities at fixed $T_{s p}$ and compared them with the coercive field measured at different $T_{s p}$ but without $J$. Results are described in the Appendix. The obtained temperature is $T_{\text {pulse }}=T_{s p}+A J^{2}[A=5.3 \times$ $\left.10^{-3} \mathrm{~K}\left(\mathrm{GA} / \mathrm{m}^{2}\right)^{-1 / 2}\right]$. We observe a good match between the set of parameters extracted from the velocities with current and the Joule heating amplitude. In agreement with Ref.[18], creep parameters exhibit a strong temperature dependance shown in the Appendix as well. This proves that our model of decomposition of the different actions of current on DW dynamics is robust, and that former results that neglected the thermal dependence of creep parameters may have led to misinterpretations [14,17,24-26].

A strong efficiency of SST-like effects is demonstrated by the adjusted $H_{e q}$ values shown in Fig. 4(b). We measure a ratio $H_{e q} / J=2 \times 10^{-2} \mathrm{mT} /\left(\mathrm{GA} / \mathrm{m}^{2}\right)$ which is large if we compare with other more conventional materials (same units): $5 \times 10^{-4}$ for $\mathrm{NiFe}$ [27] and $3 \times 10^{-7}$ for FePt [28]. It is about the same value as reported at low temperature (a few $10^{-2}$ ) for $\mathrm{Pt} / \mathrm{Co} / \mathrm{Pt}$ [29]. Above $300 \mathrm{~K}, H_{e q}$ reaches the large value obtained for the archetypical $\mathrm{Pt} / \mathrm{Co} / \mathrm{AlOx}$ [30] despite its SOT nature. As expected, we observe that the $H_{e q}$ increases 

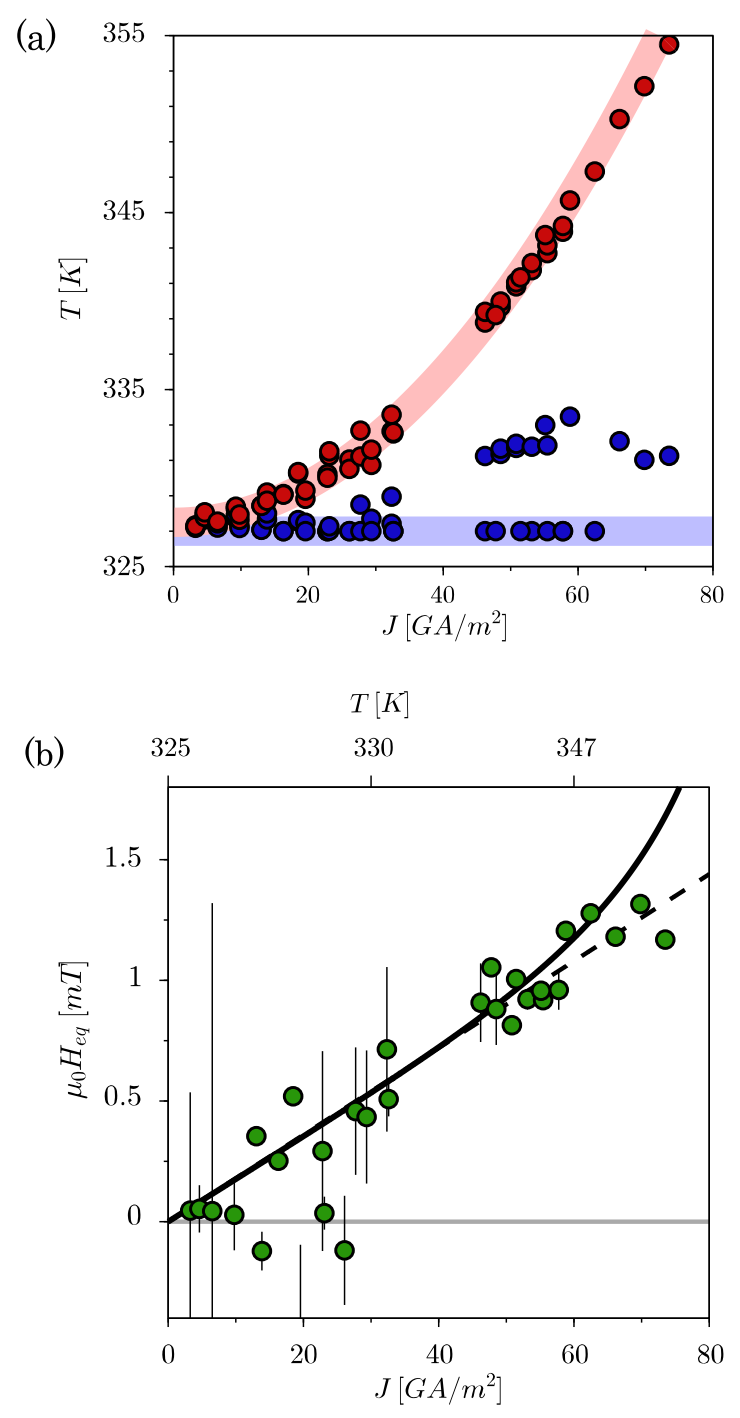

FIG. 4. (a) $T_{\text {pulse }}$ and $T_{\text {cool }}$ as a function of the current density $J$. The light red (blue) line corresponds to the Joule heating amplitude determined experimentally (the set point temperature). (b) $H_{e q}$ versus $J$ (averaged for each value of $|J|$ ). The solid line corresponds to a single behavior over the entire current range considering $u(T) / J \propto$ $P / M_{s}$ as determined in Fig. 1. The dotted line corresponds to the situation where $P / M_{s}$ is constant. The current (bottom axis) and temperature (top axis) are related by using the Joule heating amplitude.

with current. Despite the values of $H_{e q}$ being extracted from fits of DW velocity for many values of field and derived from the same range of DW velocities, the accuracy of our $H_{e q}$ data is worse at low current. In this case, $T_{\text {pulse }} \approx T_{\text {cool }}$ and the measurement is dominated by the nine-times-longer period between pulses that carries no information on $H_{e q}$.

To understand the evolution of $H_{e q}$, we propose to extend the common 1D $q-\varphi$ model to describe the pinned DW. Indeed, in the creep regime, the DW is blocked in a pinned configuration most of the time, and the macroscopic DW velocity is mostly determined by the (thermal) depinning rate. This rate is a function of the effective energy barrier, itself a function of the final DW configuration resulting from the applied forces. In the 1D model [6], the energy barrier will be determined by the final DW position in an introduced energy well [28,31-33]. In this situation, the DW settles at a position in the well that depends on both field and current. Considering these final positions, it is possible to extract a field equivalent to the current action on a pinned DW. More details can be found in Ref. [23]. As expected, $H_{e q}$ is proportional to the drift speed $u$ (so to the ratio $J P / M_{s}$ ) but, interestingly, two different behavior of pinned DW are predicted: a tilted-magnetization DW (at low current) and a DW in precession, for high $u$ above a Walker-like threshold $u_{W}^{\text {pin }}$ :

$$
u_{W}^{\mathrm{pin}}=\frac{1}{2} H_{K} \gamma \Delta .
$$

The $H_{e q}$ accounting for STT is given by Eq. (6) for the steady tilted regime $\left(u<u_{W}^{\mathrm{pin}}\right)$ and Eq. (7) for the precessional regime $\left(u>u_{W}^{\text {pin }}\right)$ :

$$
\begin{gathered}
H_{e q}^{<W}=\frac{1}{\gamma \Delta} \beta u \quad \text { for } u<u_{W}^{\mathrm{pin}}, \\
H_{e q}^{>W}=\frac{1}{\gamma \Delta}\left(\beta+\frac{1}{\alpha}\right) u \quad \text { for } u>u_{W}^{\mathrm{pin}},
\end{gathered}
$$

where $\gamma$ is the gyromagnetic ratio, $\Delta$ is the width of DW, $\alpha$ and $\beta$ are adiabatic and nonadiabatic factors of the LandauLifshitz-Gilbert equation, and $H_{K}$ is the anisotropy field between Bloch and Néel DW. These two dynamic regimes do not exist in the creep under field, and only current can induce precession of a pinned or creeping DW. Indeed, a DW that precesses continuously, but does not move, dissipates energy. As such, this precession cannot be induced by a conservative torque like the one produced by a static magnetic field whereas it can by the adiabatic spin-transfer torque.

The idea that the angular degree of freedom of the DW structure affects the depinning and the creep regimes was already discussed in similar systems and for different theoretical approaches $[31,34,35]$. Bistable transitions or quadratic adiabatic contribution were predicted and a quadratic contribution was evinced once in the depinning barrier from a single defect [36]. A quadratic adiabatic contribution, being symmetric for $\pm J$, does not affect $H_{e q}$ in our model [Eq. (4)], but instead would induce an overestimation of $T_{\text {pulse }}$. Note that the quadratic contribution is only relevant in the steady (not precessing) regime.

Considering the thermal dependance of $M_{s}$ and $P$ (Fig. 1) and using the correspondence between $J$ and $T$, we fit approximately the experimental values of $H_{e q}$ [line in Fig. 4(b)] with a $H_{e q}[\mathrm{mT}]=0.02 \mathrm{~J}\left[\mathrm{GA} / \mathrm{m}^{2}\right]$. The overall increasing trend of $H_{e q}(J)$ is reproduced over the whole range of current density. If the thermal evolution of $P / M_{S}$ is not taken into account, the dotted line corresponding to a constant $P / M_{S}$ ratio could reproduce the data as well. Let us discuss these points in regard to the predictions of our theoretical model. In low magnetization systems, such as ours, the anisotropy field is small: $H_{K} \approx 0.25 M_{s}$. Assuming $\Delta=5 \mathrm{~nm}$ from independent measurements and $P=0.5$, we can estimate $u_{W}$ to occur for $J \approx 30 \mathrm{GA} / \mathrm{m}^{2}$. It is compatible with our observations but it is difficult to detect any different evolutions of $H_{e q}$ separated by a critical current, perhaps due to the large dispersion of $H_{e q}$ data at low current.

In this study, we observe the effect of current in DWM in $\mathrm{TbFe}$ ferrimagnetic alloys in the creep regime known as the 
archetype of thermally activated regimes. We first propose a robust analysis of velocity versus field and current that allows us to separate the effect of STT from those of Joule heating. Being in the creep regime is in fact an advantage to analyze DWM. Indeed, this nonlinear speed law is very sensitive to thermal and field variations and highlights potential current effects. We observe a large modification of creep dynamics parameters, which can be attributed to Joule heating and to the strong temperature dependence of the material parameters. STT is quantified as an equivalent field $H_{e q}$. Its large strength is a consequence of the $P / M_{s}$ properties of the ferrimagnetic alloy. Finally, we predict a discontinuous current dependance of the equivalent field. This would be a signature of the existence of two propagation modes separated by a field-independent threshold current, associated with dynamic changes of the internal structure of the DW. Due to the lack of accuracy of the low-current data, we cannot evince both regimes. Their observation would be a new proof that the internal DW magnetization is relevant even in creep. This creep precessional regime should exist also in ferromagnets; however, it is harder to reach the critical current $J_{W}^{\mathrm{pin}}\left(\propto M_{s}^{2}\right)$ in materials with high $M_{s}$.

\section{APPENDIX: HEATING PHENOMENA}

As expected and observed, current injection induces strong Joule heating in our magnetic wire. As explained in the main text, this increase in temperature has a major impact in the thermal activated creep regime. To characterize Joule heating, we have determined two features: its time dependence and its amplitude.
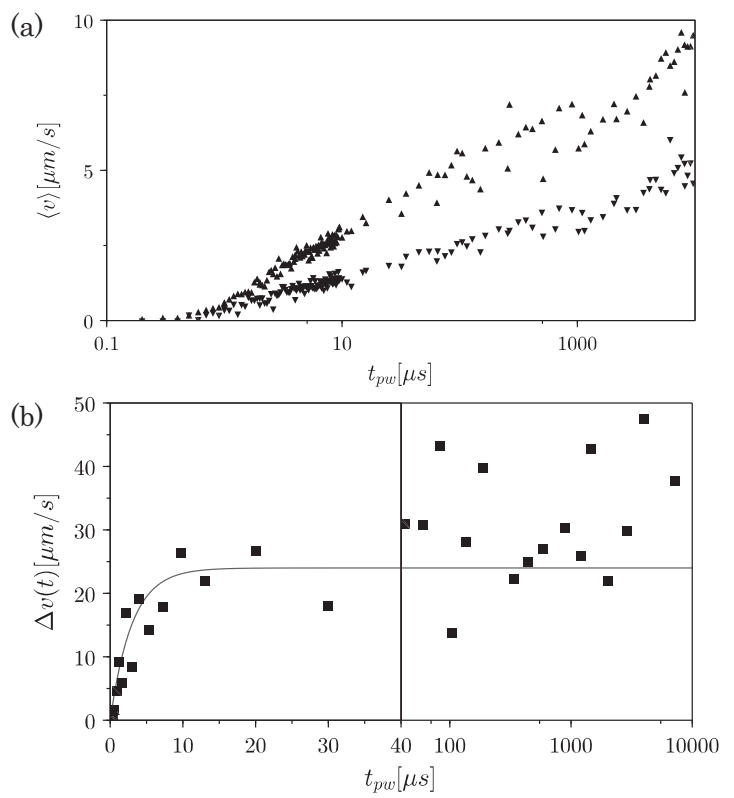

FIG. 5. (a) Evolution of the velocity of fast and slow DWs ( $v_{\text {fast }}$ up triangles, $v_{\text {slow }}$ down triangles) as a function of $t_{p w}$. (b) Instantaneous velocity difference used to establish the heating time; note the change in the timescale (linear vs log).

\section{Heating: Time Profile}

Current-injection experiments have been realized with a temperature set point $T_{s p}=328 \mathrm{~K}$ and current pulses of width $t_{p w}$ with a period $t_{p p}$. With our actual setup, we just have access to the mean speed $\langle v\rangle$ after many current pulses. The idea is to get the time profile of the temperature $T_{J}(t)$. To do so, we investigated $\langle v\rangle$ for different pulses durations. In the mean velocity [Eq. (A1)], we distinguish the velocity $v_{\text {pulse }}$ during the current pulse $t_{p w}$ (with an additional field $H_{e q}$ and modified creep parameters, see main text) from the velocity $v_{\text {cool }}$ during the cooling time $t_{p p}-t_{p w}$. It is possible to write this measured speed as

$$
\begin{aligned}
\langle v\rangle(H, \pm J)= & \frac{1}{t_{p p}} \int_{0}^{t_{p p}} v(H, \pm J(t), T(t)) d t \\
= & \frac{1}{t_{p p}} \overbrace{\int_{0}^{t_{p w}} v(H, \pm J, T(t)) d t}^{v_{\text {pulse }}} \\
& +\frac{1}{t_{p p}} \overbrace{\int_{t_{p w}}^{t_{p p}} v(H, 0, T(t)) d t}^{v_{\text {cool }}} .
\end{aligned}
$$

One way to determine the heating time is to analyze the mean velocity of fast and slow DWs as a function of $t_{p w}$ [Fig. 5(a)]. Taking the difference $\Delta v(t)$ between $v_{\text {fast }}$ and $v_{\text {slow }}$ allows us to remove the $v_{\text {cool }}$ contribution. If we differentiate
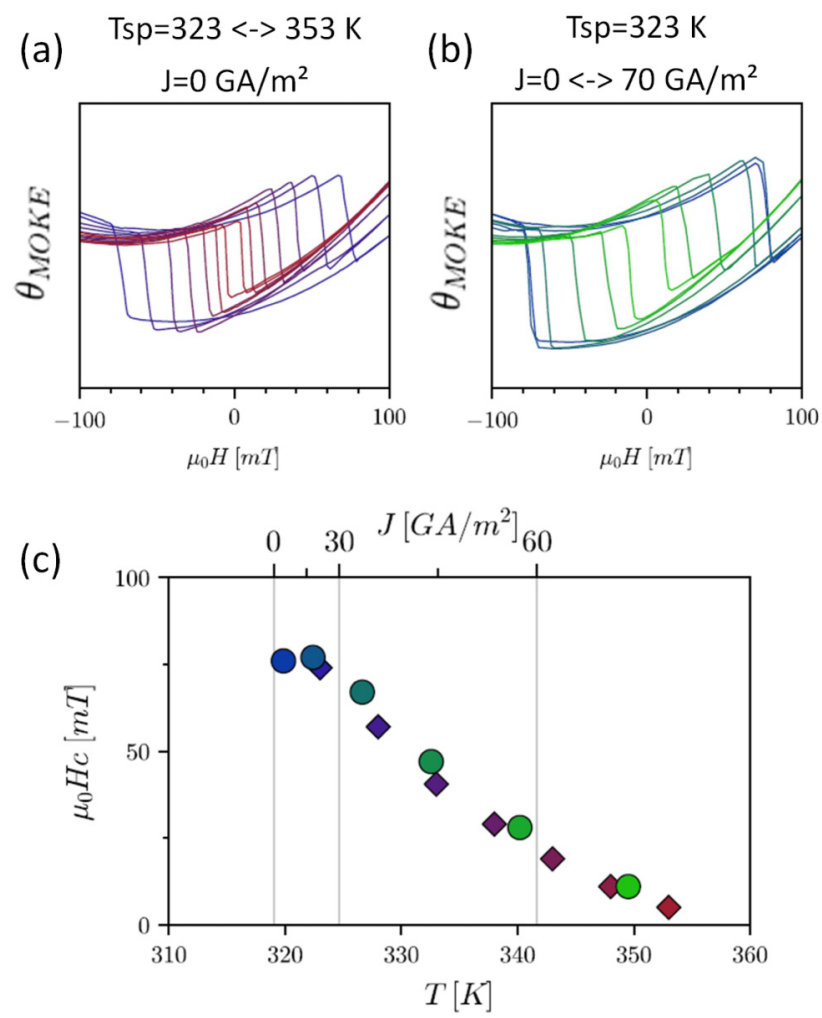

FIG. 6. (a) Evolution of MOKE hysteresis loops without current at increasing temperatures and at fixed set-point temperature with increasing current density. (b) Coercive fields as a function of temperature (bottom scale) and current density (top scale) allowing us to determine the relation between $T, T_{s p}$ and $J$. 


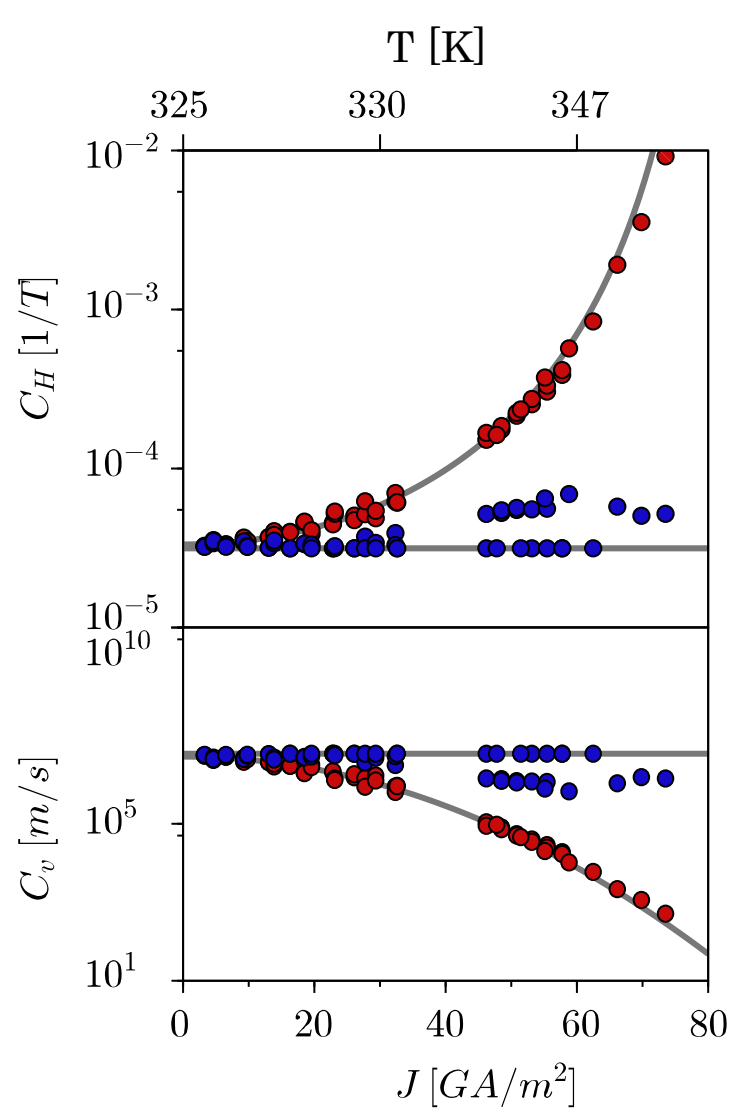

FIG. 7. Creep parameters $C_{H}$ and $C_{V}$ as a function of current density (bottom axis) and temperature (top axis) calculated from the $T_{\text {pulse }}$ temperatures extracted from the simultaneous fit of fast and slow DWs velocities. The lines represent $C_{H}\left(T_{s p}, J=0\right)$ and $C_{V}\left(T_{s p}, J=0\right)$ determined without current for different $T_{s p}$ shown in Fig. 2.

$\Delta v$ as a function of $t_{p w}$, we get the instantaneous velocity difference $\Delta v(t)$ [Eq. (A2)]. For a fixed $H$ and $J, \Delta v(t)$ only depends on $T$ and is monotonic in $T$. Therefore, its time profile is approximately the same as that of $T(t)$. When $\Delta v$ no longer changes with $t$, it implies that the temperature does not change as well [Fig. 5(b)]. It is important to note that we used a constant duty cycle (here $t_{p w} / t_{p p}=10 \%$ ), so that the temperature at the beginning of every pulse is the same and does not vary with the pulse width $t_{p w}$ [37]:

$$
\begin{aligned}
\Delta\langle v\rangle & =\langle v\rangle(H,+J)-\langle v\rangle(H,-J) \\
\Delta v(t) & =\frac{\partial \Delta\langle v\rangle}{\partial t_{p w}}=v\left(H,+J, T\left(t_{p w}\right)\right)-v\left(H,-J, T\left(t_{p w}\right)\right) .
\end{aligned}
$$

Then, we can see that the heating time is about $2.5 \mu \mathrm{s}$ [Fig. 5(b)]. This characteristic time is shorter than the used $t_{p w}$, which is why it is possible to consider a square evolution of temperature, with a constant $T_{\text {pulse }}$ temperature during the current pulse and the $T_{\text {cool }}$ during the rest of time.

\section{Heating: Amplitude}

To now measure the amplitude of Joule heating in the wire vs current density, we measured the field hysteresis loops for different current densities at a fixed $T_{s p}$ [Fig. 6(a)]. We also measured the field hysteresis loops at different temperature set points without $J$ [Fig. 6(b)]. Finally, we superimposed the coercive fields of these two sets of loops [Fig. 6(c)], considering $T_{J}=T_{s p}+A J^{2}$. The best match is obtained for $A=5.3 \times 10^{-3} \mathrm{~K}\left(\mathrm{GA} / \mathrm{m}^{2}\right)^{-1 / 2}$. With this approach, we have a bijective relation between current and heating.

\section{Creep Parameters during Current Pulses}

From the simultaneous fits of fast and slow velocities, the temperature $T_{\text {pulse }}$ and $T_{\text {cool }}$ and the STT equivalent field $H_{e q}$ were obtained. We used for that the interpolated evolution of the creep parameters $C_{H}(T)$ and $C_{V}(T)$ determined without current shown in Fig. 2 of the main text. A reversed control was done as follows: We calculated the creep parameters obtained for the $T_{\text {pulse }}$ temperatures extracted from the fits. They are shown in Fig. 7. First, the creep parameters obtained during the current pulses perfectly superimpose on their "no current" counterpart, validating the heating evaluation. Moreover, they further illustrate the thermal sensitivity of the creep parameters to temperature. From $C_{H}=\frac{1}{H_{\text {dep }}}\left(\frac{T}{T_{\text {dep }}}\right)^{4}$, a $30 \mathrm{~K}$ change obtained by $60 \mathrm{GA} / \mathrm{m}^{2}$, as in our experiments, would yield a change of only $40 \%$ in $C_{H}$ if $T_{\text {dep }}$ and $H_{\text {dep }}$ were constant, and not the observed eight-orders-of-magnitude change.
[1] D. Ralph and M. Stiles, J. Magn. Magn. Mater. 320, 1190 (2008).

[2] O. Boulle, G. Malinowski, and M. Kläui, Mater. Sci. Eng., R 72, 159 (2011).

[3] A. V. Khvalkovskiy, D. Apalkov, S. Watts, R. Chepulskii, R. S. Beach, A. Ong, X. Tang, A. Driskill-Smith, W. H. Butler, P. B. Visscher et al., J. Phys. D: Appl. Phys. 46, 074001 (2013).

[4] J.-P. Adam, S. Rohart, J.-P. Jamet, J. Ferré, A. Mougin, R. Weil, H. Bernas, and G. Faini, Phys. Rev. B 85, 214417 (2012).

[5] D. P. Bernstein, B. Bräuer, R. Kukreja, J. Stöhr, T. Hauet, J. Cucchiara, S. Mangin, J. A. Katine, T. Tyliszczak, K. W. Chou et al., Phys. Rev. B 83, 180410 (2011).

[6] A. Thiaville, Y. Nakatani, J. Miltat, and Y. Suzuki, Europhys. Lett. 69, 990 (2005).
[7] V. Jeudy, J. Curiale, J.-P. Adam, A. Thiaville, A. Lemaître, and G. Faini, J. Phys.: Condens. Matter 23, 446004 (2011).

[8] P. Hansen, C. Clausen, G. Much, M. Rosenkranz, and K. Witter, J. Appl. Phys. 66, 756 (1989).

[9] K. Buschow, Concise Encyclopedia of Magnetic and Superconducting Materials (Elsevier, 2005).

[10] T. Shirakawa, Y. Nakajima, K. Okamoto, S. Matsushita, and Y. Sakurai, in Magnetism and Magnetic Materials - 1976: Proceedings of the First Joint MMM-Intermag Conference, edited by H. C. Wolfe, J. J. Becker, and G. H. Lander, AIP Conf. Proc. No. 34 (AIP, New York, 1976), pp. 349-351.

[11] X. Jiang, L. Gao, J. Z. Sun, and S. S. P. Parkin, Phys. Rev. Lett. 97, 217202 (2006). 
[12] R. Tolley, T. Liu, Y. Xu, S. Le Gall, M. Gottwald, T. Hauet, M. Hehn, F. Montaigne, E. E. Fullerton, and S. Mangin, Appl. Phys. Lett. 106, 242403 (2015).

[13] D.-T. Ngo, K. Ikeda, and H. Awano, Appl. Phys. Express 4, 093002 (2011).

[14] S. Li, X. Liu, and A. Morisako, J. Nanosci. Nanotechnol. 12, 7550 (2012).

[15] K.-W. Moon, D.-H. Kim, S.-C. Yoo, C.-G. Cho, S. Hwang, B. Kahng, B.-C. Min, K.-H. Shin, and S.-B. Choe, Phys. Rev. Lett. 110, 107203 (2013).

[16] T. Taniguchi, K.-J. Kim, Y. Yoshimura, T. Moriyama, H. Tanigawa, T. Suzuki, E. Kariyada, and T. Ono, Appl. Phys. Express 7, 053005 (2014).

[17] S. DuttaGupta, S. Fukami, B. Kuerbanjiang, H. Sato, F. Matsukura, V. K. Lazarov, and H. Ohno, AIP Adv. 7, 055918 (2017).

[18] V. Jeudy, A. Mougin, S. Bustingorry, W. Savero Torres, J. Gorchon, A. B. Kolton, A. Lemaître, and J.-P. Jamet, Phys. Rev. Lett. 117, 057201 (2016).

[19] J. P. Pellegren, D. Lau, and V. Sokalski, Phys. Rev. Lett. 119, 027203 (2017).

[20] E. Haltz, R. Weil, J. Sampaio, A. Pointillon, O. Rousseau, K. March, N. Brun, Z. Li, E. Briand, C. Bachelet et al., Phys. Rev. Mater. 2, 104410 (2018).

[21] M. Mansuripur and M. Ruane, IEEE Trans. Magn. 22, 33 (1986).

[22] R. Schäfer, in The Handbook of Magnetism and Advanced Magnetic Materials, edited by H. Kronmüller and S. S. P. Parkin (Wiley, 2007).

[23] See Supplemental Material at http://link.aps.org/supplemental/ 10.1103/PhysRevB.99.104413 for detailed calculations of the equivalent field in the 1D model, without pinning and in the creep.
[24] L. San Emeterio Alvarez, K.-Y. Wang, S. Lepadatu, S. Landi, S. J. Bending, and C. H. Marrows, Phys. Rev. Lett. 104, 137205 (2010).

[25] K.-J. Kim, J.-C. Lee, K.-H. Shin, H.-W. Lee, and S.-B. Choe, Curr. Appl. Phys. 13, 228 (2013).

[26] J.-C. Lee, K.-J. Kim, J. Ryu, K.-W. Moon, S.-J. Yun, G.-H. Gim, K.-S. Lee, K.-H. Shin, H.-W. Lee, and S.-B. Choe, Phys. Rev. Lett. 107, 067201 (2011).

[27] N. Vernier, D. A. Allwood, D. Atkinson, M. D. Cooke, and R. P. Cowburn, Europhys. Lett. 65, 526 (2004).

[28] C. Burrowes, A. P. Mihai, D. Ravelosona, J. V. Kim, C. Chappert, L. Vila, A. Marty, Y. Samson, F. Garcia-Sanchez, L. D. Buda-Prejbeanu et al., Nat. Phys. 6, 17 (2010).

[29] O. Boulle, J. Kimling, P. Warnicke, M. Kläui, U. Rüdiger, G. Malinowski, H. J. M. Swagten, B. Koopmans, C. Ulysse, and G. Faini, Phys. Rev. Lett. 101, 216601 (2008).

[30] I. M. Miron, T. Moore, H. Szambolics, L. D. Buda-Prejbeanu, S. Auffret, B. Rodmacq, S. Pizzini, J. Vogel, M. Bonfim, A. Schuhl et al., Nat. Mater. 10, 419 (2011).

[31] V. Lecomte, S. E. Barnes, J.-P. Eckmann, and T. Giamarchi, Phys. Rev. B 80, 054413 (2009).

[32] J. Wunderlich, D. Ravelosona, C. Chappert, F. Cayssol, V. Mathet, J. Ferre, J.-P. Jamet, and A. Thiaville, IEEE Trans. Magn. 37, 2104 (2001).

[33] T. Suzuki, S. Fukami, N. Ohshima, K. Nagahara, and N. Ishiwata, J. Appl. Phys. 103, 113913 (2008).

[34] J.-V. Kim and C. Burrowes, Phys. Rev. B 80, 214424 (2009).

[35] J. Ryu, S.-B. Choe, and H.-W. Lee, Phys. Rev. B 84, 075469 (2011).

[36] K.-J. Kim, J. Ryu, G.-H. Gim, J.-C. Lee, K.-H. Shin, H.-W. Lee, and S.-B. Choe, Phys. Rev. Lett. 107, 217205 (2011).

[37] C.-Y. You, I. M. Sung, and B.-K. Joe, Appl. Phys. Lett. 89, 222513 (2006) 